\title{
Predicting CD137 upregulation on NK cells in patients receiving monoclonal antibody therapy
}

\author{
Amani Makkouk, Vandana Sundaram, Manisha Desai, Cariad Chester, Holden Maecker, Holbrook E Kohrt \\ From 30th Annual Meeting and Associated Programs of the Society for Immunotherapy of Cancer (SITC 2015) \\ National Harbor, MD, USA. 4-8 November 2015
}

\section{Background}

Monoclonal antibody (mAb) therapy-has changed the natural history of patients with B cell lymphomas, breast cancer, and head and neck cancers. However, response rates are suboptimal, highlighting the need to enhance $\mathrm{mAb}$ activity. Combining a tumor targeting $\mathrm{mAb}$ with a second antibody that activates natural killer (NK) cells may improve the therapeutic effects of mAbs. Engagement of the $\mathrm{Fc}$ receptor $(\mathrm{FcR})$ on NK cells by mAbs coating tumor cells has been previously shown to enhance their expression of CD137 [1-3]. We conducted preliminary analyses as a first step toward developing a nomogram to predict CD137 up-regulation following therapy with mAb.

\section{Methods}

Patient tumor and immune characteristics were collected from patients with Non-Hodgkin's lymphoma, breast cancer, and head and neck cancers receiving mAb therapy as part of clinical trials. CD137 expression pre- and postmAb therapy was assessed by mass cytometry time of flight (CyTOF) analysis. We examined the difference (post-pre mAB therapy) in CD137 expression for each cancer type. Specifically, we applied multivariate lasso regression tools and used classification and regression trees (CART) to predict post-CD137 expression, separately for each cancer type.

\section{Results}

We analyzed data from 62 patients with breast cancer, 46 with head/neck cancer and 91 with Non-Hodgkin's lymphoma. The difference (post-pre) in CD137 expression values was significantly different for each cancer type (mean (SD): Breast: 6.6 (6.5); Head/Neck: 11.0 (7.0); NHL: 7.5 (7.1), p

Stanford University, Stanford, CA, USA 